\title{
Oncoplastic breast surgery in asia
}

\author{
Ava Kwong* \\ Clinical Associate Professor, Chief of Breast Surgery, Assistant Dean Department \\ of Surgery, The University of Hong Kong
}

DOI: http://dx.doi.org/10.19106/JMedScieSup004804201607

\begin{abstract}
Oncoplastic breast conservation surgery has given a new light to the options of performing breast conserving surgery, by combining oncological principles of breast cancer surgery with plastic surgery techniques. The ultimate oncoplastic achievement would be the conversion of what normally would be considered as an oncologic and/or cosmetic failure when using standard techniques of breast conserving surgery into both oncological and cosmetic success and avoiding mastectomy where possible. Most techniques are fairly well established. However surgical techniques may also need adjustments based on the cohorts that the treatment is offered to. For example, for larger breasts, the technique is more forgiving. However, for smaller breast, there is still a limitation of the cosmetic outcome when a high percentage of breast volume has been excised no matter how good the technique or the surgeon is. Various aspects of oncoplastic breast surgery technique will be discussed and its application assessed among Asian Cohorts.
\end{abstract}

Keywords: breast cancer; oncoplastic breast surgery; Asia 